sterile self occupation with monotonous routines. These features may have been present for as long as the parents can remember or may have had a sudden or gradual onset at any time in the first few years of life.

Asperger's syndrome is recognised by a stilted, eccentric use of speech and problems in understanding abstractions and metaphors, accompanied by pronounced social difficulties. These children may initiate social contact but they lack empathy and respect for the feelings of others, do not adapt to their interests, and are, understandably, short of friends. They seem socially odd and characteristically have rigid, sterile interests, such as electrical circuitry or bus routes, which cannot be shared with others. Clumsiness and an odd loping gait are common associated findings.

The relations between autism, Asperger's syndrome, and specific language impairment are still controversial, and the boundaries between them are not always clear. ${ }^{3}$ Selective (or elective) mutism, however, is a distinct entity. ${ }^{4}$ In contrast to these other disorders, it is commoner in girls. The child can talk and may do so freely at home but remains silent elsewhere. There may be an underlying speech and language impairment or mild learning difficulties, but the principal mechanism is usually an anxious avoidance of speech. Older children who refuse to speak are usually doing so out of anger.

All these conditions potentially retard development and educational progress, and any suspicion of them is an indication for a detailed assessment by a paediatrician or child psychiatrist. $^{5}$

D M B HALL Consultant in Community Child Health

P HILL Senior Lecturer in Child and Adolescent Psychiatry

St George's Hospital and Medical School.

London SW17 0QT

1 Hall DMB, Hill P, Elliman D. The child surveillance handbook. Oxford: Radcliffe Medical Press, 1990 .

Kazdin AE. Childhood depression. 7 Child Psychol Psychiatry 1990;31:121-60.

3 Green J. Is Asperger's a syndrome? Dev Med Child Neurol 1990;32:743-7.

4 Rutter M, Lord C. Language disorders associated with psychiatric disturbance. In: Yule W, Rutter M, eds. Language development and disorders. Oxford: MacKeith Press, 1987:206-33.

5 Byers-Brown B. Early identification of the language disordered child. In: Martin JM, Fletcher $\mathbf{P}$ Grunwell P, Hall DMB, eds. Proceedings of the first international symposium on specific speech and language disorders. London: Association for All Speech Impaired Children, 1987:135-44.

\title{
Suicide in pregnancy and the puerperium
}

\section{Much rarer now: thanks to contraception, legal abortion, and less punitive attitudes}

Retrospective studies of suicides suggest that at least $90 \%$ of victims were psychiatrically ill at the time of their death,,$^{12}$ and depression, schizophrenia, and alcohol dependence are all known to be associated with a high risk of self destruction. Such facts may lead psychiatrists and other doctors to assume that suicide risk is determined largely by mental state and psychiatric history. In reality, social factors are often of equal or greater importance, and nothing shows this more clearly than the effect of pregnancy and childbirth on suicide rates.

Using data from the Report on Confidential Enquiries Into Maternal Deaths from 1973 to 1984 Appleby has shown that in England and Wales the standardised mortality ratio for suicide - that is, the risk relative to that in other women of the same age - is only 0.17 in the first 12 months after childbirth and a mere 0.05 in pregnancy (p 137). Although he may have missed a few suicides in the early weeks of pregnancy and in the seventh to twelfth months after delivery, such losses are unlikely to account for these low mortality ratios. Indeed, Appleby's overall suicide rate in the puerperium is somewhat higher than that reported by previous authors. Barno found only $7 \cdot 7$ suicides per million births in the first postpartum year in Minnesota, ${ }^{4}$ and Kleiner and Greston only 5.2 suicides per million births in England and Wales in 1968-75. Appleby's 76 puerperium suicides represent $9 \cdot 9$ per million births.

Very low suicide rates in pregnancy, on the other hand, are a comparatively recent phenomenon. Kleiner and Greston compared 13 studies from several countries based on the years 1900-47 with 15 studies based on the years 1943-80. In the first period $12 \cdot 6 \%$ of all women of childbearing age who committed suicide were pregnant; in the second only $1.8 \%$ were pregnant. Weir was able to identify 66 pregnant women who had committed suicide, representing 59 per million live births, by searching the records of London coroners from 1943 to $1962 .{ }^{6}$ Over half died in the third to fifth months of pregnancy and a quarter in the third month. Sixty two per cent of women (and $82 \%$ of those dying in the third month) had illegitimate pregnancies, compared with $13 \%$ of all pregnant women. A generation later Appleby could find only 14 suicides in 12 years in the whole of England and Wales. This corresponds to 1.9 suicides per million births, just $3 \%$ of Weir's figure.

The reason for the low suicide rate in pregnancy and the puerperium is almost certainly, as Appleby suggests, that motherhood and the imperative social role this entails militate strongly against self destruction. ${ }^{3}$ The much higher standardised mortality ratio he found after stillbirth (1.05), and the fact that all four of the women who committed suicide after stillbirths were primiparous, is consistent with this explanation, as is the long established finding that the suicide rate of married women is lower in those with children than in those without. ${ }^{7}$ The virtual elimination of suicide in pregnancy over the past two generations is almost certainly due to a series of social changes that have greatly reduced the number of unwanted pregnancies and their adverse societal consequences - the increasing availability of effective contraception and of legal termination of unwanted pregnancies and the decreasing stigma attached to giving birth to children outside marriage. A high proportion of Weir's pregnant women, most of whom died before these changes occurred, had conceived outside marriage and took their lives in the third month of pregnancy, the stage at which two successive menstrual bleeds would have been missed. Attempted suicide used to peak in the third month for the same reason. ${ }^{8}$ Even now Appleby finds a significantly higher suicide rate in pregnant teenagers than in older women.

Despite the dominant role of social factors the influence of psychiatric illness is also evident, at least in the puerperium. The incidence of psychosis rises sharply in the first month after childbirth, with a risk relative to that in the year before the onset of pregnancy of over $20 .^{9}$ All of Barno's puerperal suicides occurred in the first three months after delivery and all 10 women were mentally ill. ${ }^{4}$ Eighteen of Appleby's 76 suicides occurred in the first four weeks after delivery. Although he had no information about the mental health of his subjects, the fact that so high a proportion killed 
themselves in the month after childbirth and did so by violent means suggests that many of them were psychotic.

Suicide is always a tragic event, particularly if the victim is-like Shakespeare's Lucretia or Ibsen's Hedda Gabler-a young woman with an unborn or newborn child. We can be thankful that suicides of this kind are now so rare. We can also derive some satisfaction from our ability to make sense of the trends and relations we observe.

R E KENDELL

Professor of Psychiatry,

Royal Edinburgh Hospital,

Edinburgh EH $105 \mathrm{HF}$
1 Robins E, Murphy G, Wilkinson RH, Gassner S, Kayes J. Some clinical considerations in the prevention of suicide based on a study of 124 successful suicides. Am f Public Health 1959;49: $888-99$

2 Barraclough B, Bunch J, Nelson B, Sainsbury P. A hundred cases of suicide: clinical aspects. $B r \mathcal{F}$ sychiatry 1974;125:355-73.

3 Appleby L. Suicide during pregnancy and in the first postnatal year. BM $1991 ; 302: 137-40$.

Barno A. Criminal abortion deaths, illegitimate pregnancy deaths, and suicides in pregnancy, Minnesota 1950-1965. Am f Obstet Gynecol 1967;98:356-67.

Kleiner GJ, Greston WM. Overview of demographic and statistical factors. In: Kleiner GJ, Greston WM, eds. Suicide in pregnancy. Boston: John Wright, 1984.

6 Weir JG. Suicide during pregnancy in London 1943-62. In: Kleiner GJ, Greston WM, eds. Suicide in pregnancy. Boston: John Wright, 1984.

Durkheim E. Le suicide. Spaulding JA, Simpson G, transl. New York: Free Press, 1951.

8 Otto U. Suicidal attempts made by pregnant women under 21 years. Acta Paedopsychiatrica 1965;32:276-88.

9 Kendell RE, Chalmers JC, Platz C. Epidemiology of puerperal psychoses. Br $\mathcal{F}$ Psychiatry 1987;150:662-73.

\title{
Ovarian hyperstimulation: actual and theoretical risks
}

\author{
Minimise them by monitoring oestrogen concentration
}

Treatments for infertility that entail superovulation (the production of several mature ovarian follicles) carry a small risk of the ovarian hyperstimulation syndrome. This has been recognised since the early use of gonadotrophins to induce ovulation: by 1961, 60 cases (including two deaths) had been reported after treatment with gonadotrophin from the serum of pregnant mares. By 1967 the syndrome had been classified into six grades of ascending severity, ranging from mild (clinically undetectable and diagnosed by a urinary oestrogen excretion greater than $150 \mu \mathrm{g} / 24 \mathrm{~h}$ ) through moderate (enlarged ovaries presenting with abdominal distension and vomiting) to the most severe (fluid shifts leading to ascites, haemoconcentration, and abnormalities of coagulation). ${ }^{1}$

After an association between the syndrome and increased oestrogen excretion was recognised Karam et al reduced its incidence by monitoring oestrogen concentrations. ${ }^{2}$ Using ultrasonography to monitor treatment led to an apparent increase in the milder grades of the syndrome (regarded as clinically unimportant $)^{3}$ but a decrease in the more severe grades. ${ }^{4}$

A recent study reported that the hyperstimulation syndrome occurred in $3 \%$ of cycles when human menopausal gonadotrophin was used. ${ }^{5}$ These cycles were associated with raised serum concentrations of oestradiol and prolactin, with many small follicles present at the end of stimulation. Patients at risk were young and thin and had received small doses of gonadotrophin. Increased concentrations of progesterone, oestrone, 17-hydroxyprogesterone, testosterone, and $\Delta^{5}$ steroids were found in their serum ${ }^{1}$; these are the steroids whose concentrations are raised in normal pregnancy.

The syndrome is explained by a sudden increase in capillary permeability, especially of the ovarian vessels, which results in ovarian stromal oedema and a shift of body fluid, increasing blood viscosity. Why increased permeability occurs is unknown but it may result from prostaglandins whose synthesis has been stimulated by excess oestrogens. Renin may also have a role: produced by ovarian thecal cells it is present in increased amounts in hyperstimulated ovulatory cycles when compared with normal cycles. ${ }^{6}$ Renin activity reaches a higher mid-luteal peak in those cycles in which the syndrome occurs, and the size of this peak correlates with the severity of the syndrome. ${ }^{7}$ The association may be explained by the angiogenetic properties of the renin-angiotensin system-this angiogenesis is associated with increased capillary permeability.
The ovarian hyperstimulation syndrome should be preventable by carefully monitoring treatment cycles and keeping the oestrogen concentration to the minimum that is effective. This may be achieved by daily measurement of the total oestrogen excretion, serum oestradiol, or early morning urinary oestrogen to creatinine ratio. ${ }^{8}$ At the same time follicular development should be followed by ultrasonography. Gonadotrophins should be withheld or stopped if the oestrogen concentrations exceed a predetermined limit or excess multiple follicles are present. If follicular stimulation is part of a programme of assisted reproduction then further intervention-such as aspirating the follicle-is possible.

How should the hyperstimulation syndrome be managed? In mild and moderate cases simple observation of the patient is sufficient. Severe cases require prompt treatment, mainly by correcting fluid and electrolyte imbalance. Anticoagulation may be necessary, and aspiration of fluid from pleural and peritoneal cavities under ultrasonographic control has been recommended. Indomethacin may be useful (presumably because of its effect on prostaglandin synthesis). ${ }^{1}$ Inhibiting prostaglandin synthesis could, however, lead to renal failure as renal perfusion in the presence of high renin activity is preserved by the simultaneous release of vasodilatory prostaglandins. ${ }^{9}$ Laparotomy should be avoided when possible as attempted removal of the cyst may lead to massive haemorrhage. Removal may be necessary, however, when there is torsion of an ovarian cyst or intraperitoneal haemorrhage due to rupture. In these cases surgery should be as limited as possible to minimise risks and preserve precious gonadal tissue.

Could hyperstimulation increase the risk of ovarian cancer as has been recently suggested ${ }^{1011}$ Proving an association between the two will be difficult because of the likely rarity of the complication and the long time that may elapse between treatment and the clinical appearance of cancer. A retrospective case-control study would therefore answer the question sooner than a long term cohort study. Index cases might come from two sources: cases of the ovarian hyperstimulation syndrome notified to the recently created Human Fertilisation and Embryology Authority or cases of the syndrome reported as "adverse drug reactions" to the Committee on Safety of Medicines. Both would require a high level of cooperation from doctors.

Currently the risk of cancer is unproved. The risk of the hyperstimulation syndrome after superovulation is well estab- 